\title{
OPEN Early prediction of live birth for assisted reproductive technology patients: a convenient and practical prediction model
}

\author{
Hong Gao ${ }^{1,2}$, Dong-e Liu ${ }^{3}$, Yumei $\mathrm{Li}^{3}$, Xinrui Wu² \& Hongzhuan $\operatorname{Tan}^{2 \bowtie}$
}

Live birth is the most important concern for assisted reproductive technology (ART) patients. Therefore, in the medical reproductive centre, obstetricians often need to answer the following question: "What are the chances that I will have a healthy baby after ART treatment?" To date, our obstetricians have no reference on which to base the answer to this question. Our research aimed to solve this problem by establishing prediction models of live birth for ART patients. Between January 1, 2010, and May 1, 2017, we conducted a retrospective cohort study of women undergoing ART treatment at the Reproductive Medicine Centre, Xiangya Hospital of Central South University, Hunan, China. The birth of at least one live-born baby per initiated cycle or embryo transfer procedure was defined as a live birth, and all other pregnancy outcomes were classified as no live birth. A live birth prediction model was established by stepwise multivariate logistic regression. All eligible subjects were randomly allocated to two groups: group 1 ( $80 \%$ of subjects) for the establishment of the prediction models and group 2 ( $20 \%$ of subjects) for the validation of the established prediction models. The sensitivity, specificity, positive predictive value (PPV), and negative predictive value (NPV) of each prediction model at different cut-off values were calculated. The prediction model of live birth included nine variables. The area under the ROC curve was 0.743 in the validation group. The sensitivity, specificity, PPV, and NPV of the established model ranged from 97.9-24.8\%, 7.2-96.3\%, 44.8-83.8\% and $81.7-62.5 \%$, respectively, at different cut-off values. A stable, reliable, convenient, and satisfactory prediction model for live birth by ART patients was established and validated, and this model could be a useful tool for obstetricians to predict the live rate of ART patients. Meanwhile, it is also a reference for obstetricians to create good conditions for infertility patients in preparation for pregnancy.

Live birth is the most important concern for assisted reproductive technology (ART) patients; additionally, it is the only criterion used to determine whether ART treatment is successful. In recent years, ART in humans has developed very quickly, but the live birth rate is still less than optimal ${ }^{1}$. Fundamentally, numerous key factors play important roles in live birth outcomes, including embryo parameters ${ }^{2,3}$, reproductive hormone levels ${ }^{4-6}$, and the patient's age ${ }^{7,8}$, among others; however, the degrees to which these factors affect birth outcomes are not clear. Consequently, it is very significant to investigate how these factors affect live birth outcomes, determine their degrees of influence and then establish live birth prediction models.

Previous studies have used various strategies to predict live birth for different sub-groups of ART patient, including in vitro fertilization (IVF) patients ${ }^{9}$, intracytoplasmic sperm injection (ICSI) patients ${ }^{10}$, ICSI patients with uncompromised ovarian reserve ${ }^{11}$, and poor ovarian responders ${ }^{12,13}$. Different strategies have included risk scoring systems ${ }^{14}$ deep phenotyping ${ }^{9}$, granulosa cell biomarkers ${ }^{1}$, and clinical characteristics (female age, testicular sperm extraction cycle, male and female reproductive hormones, spermatozoa parameters, infertility diagnosis, and oocyte parameters $)^{10,11,15}$. The sensitivity and specificity of these live birth prediction models exhibit large variations. Prajna Banerjee et al..$^{9}$ used 52 clinical characteristics to establish a model. The area under the receiver operating characteristic (ROC) curve was up to 0.80 for IVF patients in their first cycles, but in their

\footnotetext{
${ }^{1}$ School of Nursing, University of South China, Hengyang 421001, Hunan, China. ${ }^{2}$ Department of Epidemiology and Health Statistics, XiangYa School of Public Health, Central South University, Changsha 410008, Hunan, China. ${ }^{3}$ Reproductive Medicine Centre, Xiangya Hospital of Central South University, Changsha 410008, Hunan, China. ${ }^{\boxplus}$ email: tanhz99@qq.com
} 


\begin{tabular}{|c|c|c|c|}
\hline Variables & $\begin{array}{l}\text { Live birth } \\
\mathrm{n}=6012\end{array}$ & $\begin{array}{l}\text { No live birth } \\
\mathrm{n}=7814\end{array}$ & $p$-value \\
\hline \multicolumn{4}{|l|}{ Pre-ART factors } \\
\hline Maternal age, year & $30.63 \pm 4.36$ & $32.67 \pm 5.50$ & $<0.001$ \\
\hline Body mass index, $\mathrm{kg} / \mathrm{m}^{2}$ & $21.61 \pm 2.97$ & $21.97 \pm 3.05$ & $<0.001$ \\
\hline Uterine volume, $\mathrm{mL}$ & $52.21 \pm 22.37$ & $54.42 \pm 25.83$ & $<0.001$ \\
\hline Female infertility duration, year & $4.67 \pm 3.31$ & $5.42 \pm 3.98$ & $<0.001$ \\
\hline No. of previous pregnancies & $1.07 \pm 1.32$ & $1.27 \pm 1.46$ & $<0.001$ \\
\hline No. of abortions & $0.62 \pm 0.97$ & $0.78 \pm 1.13$ & $<0.001$ \\
\hline Basal FSH ${ }^{\mathrm{a}}, \mathrm{mIU} / \mathrm{mL}$ & $6.79 \pm 18.60$ & $7.28 \pm 7.49$ & $<0.001$ \\
\hline Number of previous ART treatments & $1.72 \pm 0.99$ & $2.01 \pm 1.18$ & $<0.001$ \\
\hline \multicolumn{4}{|l|}{ Protocol and treatment factors } \\
\hline Total dose of gonadotropin, IU & $2007.61 \pm 841.34$ & $2049.03 \pm 1074.933$ & $<0.001$ \\
\hline No. of antral follicles & $6.25 \pm 3.29$ & $5.50 \pm 3.33$ & $<0.001$ \\
\hline Total no. of oocytes & $11.01 \pm 4.50$ & $9.12 \pm 4.66$ & $<0.001$ \\
\hline Sperm concentration, million $/ \mathrm{mL}$ & $109.90 \pm 98.18$ & $104.73 \pm 92.83$ & 0.04 \\
\hline Sperm viability, $\%$ & $41.28 \pm 22.31$ & $41.38 \pm 22.14$ & 0.87 \\
\hline Sperm progressive motility, $\%$ & $34.82 \pm 23.33$ & $34.87 \pm 23.54$ & 0.68 \\
\hline Endometrial thickness before embryo transfer, $\mathrm{mm}$ & $10.51 \pm 2.10$ & $9.99 \pm 2.11$ & $<0.001$ \\
\hline Total no. of transferred embryos & $1.98 \pm 0.27$ & $1.88 \pm 0.45$ & $<0.001$ \\
\hline
\end{tabular}

Table 1. Comparison of the characteristics between the live birth and no live birth groups comprising 13,826 ART patients from 2010-2017 (continuous variables). (Mean \pm SD). ${ }^{a} \mathrm{FSH}$, follicle-stimulating hormone.

subsequent treatments, this value decreased to less than 0.68 . Although many live birth prediction models have been established, they are rarely used in clinical practice. The main reasons may include the following: (1) they cannot be applied to all ART patients because the model is based on only 1-2 types of ART patients; (2) some predictors need more complicated and expensive laboratory tests; (3) the use of these models is not sufficiently convenient, and (4) some models are less accurate than others for predicting live birth.

We aimed to establish a convenient and practical live birth prediction model that has higher predictive value and that can be applied to all ART patients.

\section{Results}

There were 15,717 ART treatments performed from 2012 to 2017. Of these, 1891 subjects who had missing information on their live birth outcomes were excluded, leaving 13,826 subjects for analysis. Among them, $80 \%$ of the subjects $(11,071)$ were allocated to group 1 (establishment model), and $20 \%$ of them (2755) were allocated to group 2 (validation model).

Univariate analysis results. We analysed the relationships between the variables and live birth outcomes by univariate analysis. Twenty-two variables were found to be significantly associated with live birth outcomes $(p$-value $<0.05$, Tables 1,2$)$.

Logistic regression analysis and prediction model establishment. Based on our univariate analysis results, we found that maternal age, body mass index, number of previous ART treatments, female infertility duration, number of previous pregnancies, number of abortions, basal FSH, sperm concentration, endometrial thickness before embryo transfer, number of antral follicles, total number of oocytes, sperm viability, sperm progressive motility, type of embryo transfer, quality of transferred embryos, maternal education, infertility diagnosis, uterine volume, artificial insemination technology used, stimulation protocol, total number of transferred embryos, and total dose of gonadotropin were significantly associated with live birth. We used these variables as independent variables to perform multiple logistic regression analysis. The variables are presented in Table 3 . We found there was not multicollinearity among those variables (Tol $>0.1$, VIF $<10$, shown in Table 4). Likelihood ratio forward stepwise method ( $\alpha=0.05$ for entry, and $\alpha=0.10$ for removal) was used in the logistic regression. Finally, the prediction model of live birth was established, including nine variables (shown in Table 4). Each step of this model is shown in Supplementary Table S1. The area under the ROC curve was 0.722 (95\% CI, 0.709-0.735). The model is as follows: Logit $\mathrm{P}=-1.857+0.199 \mathrm{X}_{1}+0.150 \mathrm{X}_{2}+0.276 \mathrm{X}_{3}+0.077 \mathrm{X}_{5}-0.149 \mathrm{X}_{8}+1.20$ $5 \mathrm{X}_{9}+0.690 \mathrm{X}_{12}+0.770 \mathrm{X}_{13}+0.534 \mathrm{X}_{19}$

Verification prediction model. We performed a validation of the prediction model of live birth by using the model in the validation group [with $20 \%$ of the subjects (2755)]. The area under the ROC curve was 0.743 (95\% CI, 0.719-0.768) (Fig. 1). Table 5 shows the practical predictive value for live birth with different cut-off values. The sensitivity(SE) and specificity(SP) ranged from $97.9-24.8 \%$ and $7.2-96.3 \%$, respectively, at different cut-off values. 


\begin{tabular}{|c|c|c|c|c|}
\hline Variables & $\mathbf{N}$ & Live birth (n) & Live birth rate (\%) & $p$-value \\
\hline \multicolumn{5}{|l|}{ Pre-ART factors } \\
\hline Maternal education & & & & $<0.001$ \\
\hline Under 6 years & 1086 & 392 & 36.1 & \\
\hline $6-9$ years & 6186 & 2723 & 44.0 & \\
\hline $10-12$ years & 1737 & 834 & 48.0 & \\
\hline 13 years and over & 4341 & 2047 & 47.2 & \\
\hline Infertility diagnosis & & & & $<0.001$ \\
\hline Male factors & 1890 & 818 & 43.3 & \\
\hline Ovulation dysfunction & 58 & 25 & 43.1 & \\
\hline Decreased ovarian reserve & 150 & 36 & 24.0 & \\
\hline Tubal factors & 9568 & 4164 & 43.5 & \\
\hline Uterine factors & 142 & 54 & 38.0 & \\
\hline Chromosome abnormality & 14 & 5 & 35.7 & \\
\hline Unexplained factors & 44 & 15 & 34.1 & \\
\hline Male + female factors & 1700 & 774 & 45.5 & \\
\hline \multicolumn{5}{|l|}{ Protocol and treatment factors } \\
\hline Stimulation protocol & & & & $<0.001$ \\
\hline Long protocol & 5648 & 3024 & 53.5 & \\
\hline Short protocol & 1752 & 521 & 29.7 & \\
\hline Other protocol & 2703 & 752 & 27.8 & \\
\hline Artificial insemination technology & & & & 0.02 \\
\hline IVF & 9820 & 4344 & 44.2 & \\
\hline ICSI & 2871 & 1215 & 42.3 & \\
\hline IVF + ICSI & 884 & 358 & 40.5 & \\
\hline IUI $^{\mathrm{a}}$ & 77 & 27 & 35.1 & \\
\hline Type of embryo transfer & & & & $<0.001$ \\
\hline Fresh embryo & 7431 & 3544 & 47.7 & \\
\hline Frozen-thawed embryo & 6309 & 2442 & 38.7 & \\
\hline Quality of the transferred embryos ${ }^{\mathrm{b}}$ & & & & $<0.001$ \\
\hline I & 11,585 & 5482 & 47.3 & \\
\hline II & 1112 & 334 & 30.0 & \\
\hline III & 443 & 62 & 14.0 & \\
\hline
\end{tabular}

Table 2. Comparison of the live birth rates of the different sub-groups of the 13,826 ART patients from 2010-2017 (categorical variables). ${ }^{\mathrm{a}}$ IUI, intrauterine insemination. ${ }^{\mathrm{b}}$ Quality of the transferred embryos: I is the best-quality embryo, followed by II and III.

\section{Discussion}

We established a prediction model of live birth by multivariable logistic regression analysis, and this model included 9 common variables. In the establishment model group, the ROC value was 0.722 , and there was good calibration. In the validation model group set, the ROC value was 0.743 , and the model was calibrated well.

Our model has several obvious advantages. Our model is a convenient and practical prediction model because information on all the variables included in the model is generally available in the clinic, and there is no need for any special test. Some predictors in our model, such as endometrial thickness, stimulation protocol, and embryo parameters, can be the focus of interventions. Therefore, the model has a certain predictive value and instructional clinical value in early treatments. The model can predict a live birth in the early pregnancy stage because information on almost all the variables included in the model is available at the beginning of pregnancy. Moreover, our model has acceptable clinical predictive value, and the area under the ROC curve reached 0.743 in the validation group, which is higher than the values of most of the previously reported models that were similar to our model ${ }^{10,13,16}$. Although the ROC values of some models are larger than ours, the variables in these models, such as gene or granulosa cell biomarkers, may be inconvenient to assess ${ }^{1,17}$. Moreover, a variable, such as gene expression, may be unchangeable and have no preventive value ${ }^{1}$. Furthermore, information on variables, such as HCG and progesterone may only be attainable after pregnancy is achieved and cannot be used for the prediction of a live birth ${ }^{9,18,19}$. Many previous prediction models of live birth are not applicable to all ART patients but are instead only applicable for a specific infertility subgroup ${ }^{10,20-22}$; however, our model is suitable for different artificial insemination technologies and all ART patients.

We have established a highly discriminatory, well-calibrated, robust, and practical prediction model that can use available clinical data to predict the live birth rate and may be transferred to corresponding computer 


\begin{tabular}{|c|c|c|}
\hline Variables & Variable names & Assignment statement \\
\hline Maternal age (year) & $\mathrm{X}_{1}$ & $1=<25,2=25 \sim, 3=30 \sim, 4=\geq 35$ \\
\hline Maternal education (year) ${ }^{\mathrm{a}}$ & $\mathrm{X}_{2}$ & $1=<10,0=\geq 10$ \\
\hline Number of previous ART treatments & $\mathrm{X}_{3}$ & $1=1,2=2,3=\geq 3$ \\
\hline Uterine volume $(\mathrm{mL})^{\mathrm{a}}$ & $\mathrm{X}_{4}$ & $1=<30,0=30 \sim, 2=50 \sim, 3=\geq 70$ \\
\hline No. of abortions & $\mathrm{X}_{5}$ & $1=0,2=1,3=2,4=\geq 3$ \\
\hline Sperm concentration (million/mL) & $\mathrm{X}_{6}$ & $1=<43.00,2=43.00 \sim, 3=85.00 \sim, 4=\geq 144.40$ \\
\hline Infertility diagnosis $^{\mathrm{a}}$ & $\mathrm{X}_{7}$ & $\begin{array}{l}1=\text { male factors, } 2=\text { ovulation dysfunction, } 3=\text { decreased ovarian reserve, } 4=\text { tubal factors, } 5=\text { uterine fac- } \\
\text { tors, } 6=\text { chromosome abnormality, } 7=\text { unexplained, } 0=\text { male }+ \text { female factors }\end{array}$ \\
\hline Endometrial thickness before embryo transfer (mm) & $\mathrm{X}_{8}$ & $1=<8,2=8 \sim, 3=10 \sim, 4=\geq 12$ \\
\hline Total no. of transferred embryos ${ }^{\mathrm{a}}$ & $\mathrm{X}_{9}$ & $1=1,0=2$ \\
\hline Total dose of gonadotropin (IU) ${ }^{\mathrm{a}}$ & $\mathrm{X}_{10}$ & $1=<1500,0=1500 \sim, 2=2000 \sim, 3=\geq 2500$ \\
\hline Total no. of oocytes & $\mathrm{X}_{11}$ & $1=<8,2=8 \sim, 3=\geq 13$ \\
\hline Quality of the transferred embryos & $\mathrm{X}_{12}$ & $1=\mathrm{I}, 2=\mathrm{II}, 3=\mathrm{III}$ \\
\hline Stimulation protocol ${ }^{\mathrm{a}}$ & $\mathrm{X}_{13}$ & $0=$ long protocol, $1=$ short protocol, $1=$ other protocol \\
\hline Sperm viability (\%) & $\mathrm{X}_{14}$ & $0=<40.00,1=\geq 40.00$ \\
\hline Sperm progressive motility (\%) & $\mathrm{X}_{15}$ & $0=<32.00,1=\geq 32.00$ \\
\hline BMI $\left(\mathrm{kg} / \mathrm{m}^{2}\right)$ & $\mathrm{X}_{16}$ & $1=<18.50,2=18.50 \sim, 3=24.00 \sim, 4=\geq 28.00$ \\
\hline Female infertility duration (yr) & $\mathrm{X}_{17}$ & $1=<3,2=3 \sim, 3=5 \sim, 4=\geq 7$ \\
\hline No. of previous pregnancies & $\mathrm{X}_{18}$ & $1=0,2=1,3=2,4=3,5=\geq 4$ \\
\hline Basal FSH (mIU/mL) & $\mathrm{X}_{19}$ & $0=<10,1=\geq 10$ \\
\hline No. of antral follicles & $\mathrm{X}_{20}$ & $0=<5,1=\geq 5$ \\
\hline Type of embryo transfer & $\mathrm{X}_{21}$ & $0=$ fresh embryo, 1 = frozen-thawed embryo \\
\hline Artificial insemination technology ${ }^{\mathrm{a}}$ & $\mathrm{X}_{22}$ & $0=\mathrm{IVF}, 1=\mathrm{ICSI}, 2=\mathrm{IVF}+\mathrm{ICSI}, 3=\mathrm{IUI}$ \\
\hline Live birth outcome & $\mathrm{Y}$ & $1=$ no live birth, $0=$ live birth \\
\hline
\end{tabular}

Table 3. Variable assignment in the multivariate logistic regression analysis. ${ }^{a} \mathrm{X}_{2}, \mathrm{X}_{4}, \mathrm{X}_{7}, \mathrm{X}_{9}, \mathrm{X}_{10}, \mathrm{X}_{13}, \mathrm{X}_{22}$ were entered into the models as dummy variables, and the group with the highest live birth rate was selected as the reference group, which was assigned "0".

\begin{tabular}{|c|c|c|c|c|c|}
\hline \multirow[b]{2}{*}{ Variables } & \multirow[b]{2}{*}{$\mathbf{B}_{0}$} & \multirow[b]{2}{*}{$b_{i}$} & \multirow[b]{2}{*}{$p$} & \multicolumn{2}{|c|}{$\begin{array}{l}\text { Collinearity } \\
\text { Statistics }\end{array}$} \\
\hline & & & & Tolerance & VIF \\
\hline Maternal age (X1) & 0.199 & 0.035 & $<0.001$ & 0.879 & 1.138 \\
\hline Maternal education $(\mathrm{X} 2)$ & 0.150 & 0.058 & 0.009 & 0.996 & 1.004 \\
\hline Number of previous ART treatments (X3) & 0.276 & 0.047 & $<0.001$ & 0.679 & 1.472 \\
\hline No. of abortions (X5) & 0.077 & 0.034 & 0.023 & 0.905 & 1.105 \\
\hline Endometrial thickness before embryo transfer (X8) & -0.149 & 0.033 & $<0.001$ & 0.881 & 1.135 \\
\hline Total no. of transferred embryos (X9) & 1.205 & 0.119 & $<0.001$ & 0.949 & 1.053 \\
\hline Quality of the transferred embryos (X12) & 0.690 & 0.071 & $<0.001$ & 0.983 & 1.018 \\
\hline Stimulation protocol (X13) & 0.770 & 0.071 & $<0.001$ & 0.654 & 1.530 \\
\hline Basal FSH (X19) & 0.534 & 0.123 & $<0.001$ & 0.954 & 1.048 \\
\hline Constant & -1.857 & 0.175 & $<0.001$ & & \\
\hline
\end{tabular}

Table 4. The association between live birth and the predictive variables included in the logistic predicting model.

software for easy operation. Clinicians and public health workers can easily use this model to identify high-risk populations for the management by ART.

As we all know, breeding a new life is a very complex process, which will be affected by many known and unknown factors. Especially, for infertility patients, the condition will be more complex and changeable. Medical technology level of different hospitals and doctors quite naturally plays a fundamental role. To date, it is hard to predict live birth rates before embryo transfer. In the early stage of infertility treatment, patients and doctors are most concerned about "How many normal oocytes are there?", "How many normal sperm are there?" and "How many embryos can be transferred?" Therefore, the prediction of live birth rate is at least based on the successful embryo transfer. However, this finding can be used as a guidance to try to create good conditions for infertility patients in preparation for pregnancy. 


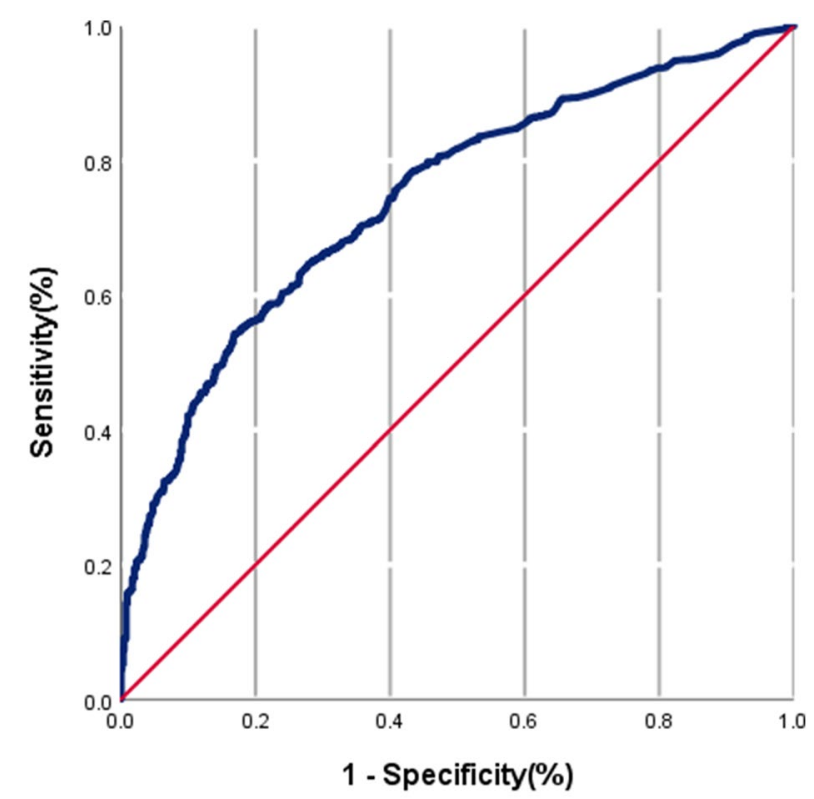

Area under the ROC curve $=\mathbf{0 . 7 4 3}$

Figure 1. Receiver operating characteristic (ROC) curve of the live birth prediction model for ART patients.

\begin{tabular}{|c|c|c|c|c|c|c|c|c|}
\hline \multirow[b]{2}{*}{ Cut-off value } & \multicolumn{4}{|c|}{\begin{tabular}{|l|} 
Group 1 \\
\end{tabular}} & \multicolumn{4}{|c|}{ Group 2} \\
\hline & SE & SP & PPVa & NPVb & SE & SP & PPV & NPV \\
\hline 0.25 & 99.7 & 1.7 & 43.9 & 88.0 & 97.9 & 7.2 & \begin{tabular}{|l|l|}
44.8 \\
\end{tabular} & 81.7 \\
\hline 0.30 & 95.5 & 14.3 & 46.2 & 80.5 & 93.8 & 20.2 & 47.5 & 80.9 \\
\hline 0.35 & 86.7 & 35.1 & 50.7 & 77.4 & 89.0 & 34.7 & 51.2 & 80.4 \\
\hline 0.40 & 78.7 & 0.50 & 54.8 & 75.3 & 84.2 & 43.9 & 53.6 & 78.3 \\
\hline 0.45 & 72.4 & 58.9 & 57.6 & 73.5 & 77.6 & 57.3 & 58.3 & 76.9 \\
\hline 0.50 & 67.8 & 66.3 & 60.8 & 72.8 & 68.3 & 65.9 & 60.7 & 73.0 \\
\hline 0.55 & 62.2 & 72.5 & 63.5 & 71.4 & 65.1 & 71.3 & 63.6 & 72.6 \\
\hline 0.60 & 55.3 & 78.9 & 66.9 & 69.6 & 57.5 & 78.7 & 67.5 & 70.6 \\
\hline 0.65 & 44.5 & 84.9 & 69.4 & 66.5 & 50.3 & 84.7 & 71.7 & 68.9 \\
\hline 0.70 & 34.4 & 90.8 & 74.2 & 64.3 & 43.1 & 89.4 & 75.8 & 67.1 \\
\hline 0.75 & 25.4 & 94.3 & 77.4 & 62.1 & 32.4 & 93.4 & 79.1 & 64.2 \\
\hline 0.80 & 18.3 & 96.5 & 80.1 & 60.5 & 24.8 & 96.3 & 83.8 & 62.5 \\
\hline
\end{tabular}

Table 5. The validity of the prediction model with different cut-off values in different groups (\%). ${ }^{a} \mathrm{PPV}$ : positive predictive value. ${ }^{b} \mathrm{NPV}$ : negative predictive value.

Our live birth prediction models were further validated with a separate sample, allowing us the ability to evaluate the true predictive performance of the models when they are being used in other populations. We also examined the impacts of different cut-off values on sensitivity, specificity, PPV and NPV, to establish an appropriate reference range. Clinicians and public health workers could conveniently select different cut-off values in their live birth assessment process to obtain optional results.

There are several limitations to this study. Our data were obtained only from a large reproductive medicine centre, and the ROC values of our model are not the largest among the reported models. Therefore, we do not think the model is unchangeable. With the development of medical technology, new variables will be added to our model, and our prediction model will be continuously optimized. In addition, the applicability of the model in other clinics needs to be further verified, which will be our next research work.

In conclusion, a prediction model for live birth by ART patients was established and validated. The model is stable, reliable, convenient, and satisfactory; furthermore, this model could be a useful tool for early-gestation predictions by obstetricians of whether or not a ART patient has a high probability of a live birth and for trying to create good conditions for ART patients in preparation for pregnancy. 


\section{Methods}

Study data. Between January 12010 and May 1 2017, we conducted a retrospective cohort study of women undergoing ART treatments at the Reproductive Medicine Centre, Xiangya Hospital of Central South University, Hunan, China. All data of the subjects were retrieved from the electronic medical records (Haitai, Nanjing, China) of Xiangya Hospital Central South University. The inclusion criteria for data analysis were as follows: (1) completion of the basic medical examination; (2) completion of the entire ART treatment cycle; (3) complete record of the couple's basic information; and (4) thorough documentation of the live birth outcome.

Outcome measures. In our study, we focused on live birth. The birth of at least one live-born baby per initiated cycle or embryo transfer procedure was defined as a live birth, and all the other adverse pregnancy outcomes were classified as no live birth.

Statistical analysis. A live birth prediction model was established by stepwise multivariate logistic regres$\operatorname{sion}(\alpha=0.05$ for entry, and $\alpha=0.10$ for removal). When establishing the model, the criteria for selecting predictive variables were as follows: first, $p$ value was less than 0.05 ; second, it contributed to improving the area under the ROC curve. All eligible subjects were randomly allocated to two groups: group 1 ( $80 \%$ of subjects) for the establishment of the prediction models and group 2 (20\% of subjects) for the validation of the established prediction models. Subjects of the two groups were independent without repetition. The areas under the ROC curve generated by the logistic regression model were applied to evaluate the effectiveness of the prediction models. We further calculated the sensitivity, specificity, positive predictive value, and negative predictive value of the prediction models with different cut-off values.

All data were managed and analysed using the statistical package for social sciences (SPSS) software version 17.0 (Chicago, IL, SPSS Inc. 2008) and Excel (Microsoft Corp., Redmond, WA, USA). Measurement data are described as the mean \pm standard deviation (SD), and enumeration data are described as numbers (percentages). All $p$ values corresponded to two-sided tests, and $p<0.05$ was considered statistically significant.

Ethical approval and consent to participate. The study was approved by the Ethics Committee of Xiangya Hospital of Central South University, mainly including the use of data from various clinical examination and laboratory tests of patients. All infertility patients presenting to the Reproductive Medicine Centre, Xiangya Hospital of Central South University, Hunan, China, who were planned for ART treatments and who signed the informed consent were enrolled in the study from January 12010 and May 12017 . In addition, we confirmed that all methods were performed in accordance with the assisted reproductive technology relevant guidelines and regulations.

\section{Data availability}

The datasets used and/or analysed during the current study are available from the corresponding author on reasonable request.

Received: 4 June 2020; Accepted: 7 December 2020

Published online: 11 January 2021

\section{References}

1. Kordus, R. J. \& LaVoie, H. A. Granulosa cell biomarkers to predict pregnancy in ART: pieces to solve the puzzle. Reproduction 153, 69-83 (2017).

2. Zhao, Y. Y., Yu, Y. \& Zhang, X. W. Overall blastocyst quality, trophectoderm grade, and inner cell mass grade predict pregnancy outcome in euploid blastocyst transfer cycles. Chin. Med. J. (Engl.) 131, 1261-1267 (2018).

3. Aparicio-Ruiz, B., Romany, L. \& Meseguer, M. Selection of preimplantation embryos using time-lapse microscopy in in vitro fertilization: state of the technology and future directions. Birth Defects Res. 110, 648-653 (2018).

4. Zhao, W. et al. Effects of oestradiol for luteal phase support in fresh embryo transfer cycles: a retrospective cohort study. Clin. Endocrinol. (Oxf.) 89, 1-8 (2018).

5. Seikkula, J. et al. Mid-luteal phase gonadotropin-releasing hormone agonist support in frozen-thawed embryo transfers during artificial cycles: a prospective interventional pilot study. J. Gynecol. Obstet. Hum. Reprod. 169, 1-5 (2018).

6. Daney, D. M. F. et al. What are the likely IVF/ICSI outcomes if there is a discrepancy between serum AMH and FSH levels? A multicenter retrospective study. J. Gynecol. Obstet. Hum. Reprod. 46, 629-635 (2017).

7. Amsiejiene, A. et al. The influence of age, body mass index, waist-to-hip ratio and anti-Mullerian hormone level on clinical pregnancy rates in ART. Gynecol. Endocrinol. 33, 41-43 (2017).

8. Srouji, S. S. et al. Predicting in vitro fertilization live birth using stimulation day 6 estradiol, age, and follicle-stimulating hormone. Fertil. Steril. 84, 795-797 (2005).

9. Banerjee, P. et al. Deep phenotyping to predict live birth outcomes in in vitro fertilization. Proc. Natl. Acad. Sci. USA 107, 1357013575 (2010).

10. Meijerink, A. M. et al. Prediction model for live birth in ICSI using testicular extracted sperm. Hum. Reprod. 31, 1942-1951 (2016).

11. Goldman, R. H. et al. Predicting the likelihood of live birth for elective oocyte cryopreservation: a counseling tool for physicians and patients. Hum. Reprod. 32, 853-859 (2017).

12. Lainas, T. G. et al. Live birth rates after modified natural cycle compared with high-dose FSH stimulation using GnRH antagonists in poor responders. Hum. Reprod. 30, 2321-2330 (2015).

13. Lehert, P. et al. Predicting live birth for poor ovarian responders: the PROsPeR concept. Reprod. Biomed. Online 37, 43-52 (2018).

14. Michailidou-Ahmed, C., Sharpe, A. A., Burrell, E. V., Blower, J. A. \& Potdar, N. HBA score in relation to donor semen profiles and live birth rates: a preliminary study. Hum. Fertil. (Camb.) 19, 289-298 (2016).

15. Peng, J., Zhang, Z., Yuan, Y., Cui, W. \& Song, W. Pregnancy and live birth rates after microsurgical vasoepididymostomy for azoospermic patients with epididymal obstruction. Hum. Reprod. 32, 284-289 (2017).

16. Dhillon, R. K. et al. Predicting the chance of live birth for women undergoing IVF: a novel pretreatment counselling tool. Hum. Reprod. 31, 84-92 (2016). 
17. Bracewell-Milnes, T. et al. Metabolomics as a tool to identify biomarkers to predict and improve outcomes in reproductive medicine: a systematic review. Hum. Reprod. Update 23, 723-736 (2017).

18. Blakemore, J. K., Kofinas, J. D., McCulloh, D. H. \& Grifo, J. Serum progesterone trend after day of transfer predicts live birth in fresh IVF cycles. J. Assist. Reprod. Genet. 34, 339-343 (2017).

19. Iliodromiti, S., Kelsey, T. W., Wu, O., Anderson, R. A. \& Nelson, S. M. The predictive accuracy of anti-Mullerian hormone for live birth after assisted conception: a systematic review and meta-analysis of the literature. Hum. Reprod. Update 20, 560-570 (2014).

20. van Loendersloot, L. L., van Wely, M., Repping, S., Bossuyt, P. M. \& van der Veen, F. Individualized decision-making in IVF: calculating the chances of pregnancy. Hum. Reprod. 28, 2972-2980 (2013).

21. Lintsen, A. M. et al. Predicting ongoing pregnancy chances after IVF and ICSI: a national prospective study. Hum. Reprod. 22, 2455-2462 (2007).

22. Alson, S., Bungum, L. J., Giwercman, A. \& Henic, E. Anti-mullerian hormone levels are associated with live birth rates in ART, but the predictive ability of anti-mullerian hormone is modest. Eur. J. Obstet. Gynecol. Reprod. Biol. 225, 199-204 (2018).

\section{Acknowledgements}

This work was supported by the Start-up Fund Project of Doctor's Introduction of Talents of University of South China in China [No. 190XQD114] and the Excellent Young Talents Fund Program of Education Department of Hunan Province in China [No. (2019)90-18B277].

\section{Author contributions}

H.G., D.E.L. and H.Z.T. formulated the study questions and directed their implementation. H.G., D.E.L. and H.Z.T. contributed to the study design. H.G. performed the statistical analysis. X.R.W. and H.Z.T. provided guidance on the statistical analysis. D.E.L. and Y.M.L. provided clinical data. H.G. and H.Z.T. drafted the article. All authors were involved in revising the article and have approved this final version.

\section{Competing interests}

The authors declare no competing interests.

\section{Additional information}

Supplementary Information is available for this paper at https://doi.org/10.1038/s41598-020-79308-9.

Correspondence and requests for materials should be addressed to H.T.

Reprints and permissions information is available at www.nature.com/reprints.

Publisher's note Springer Nature remains neutral with regard to jurisdictional claims in published maps and institutional affiliations.

(c) Open Access This article is licensed under a Creative Commons Attribution 4.0 International License, which permits use, sharing, adaptation, distribution and reproduction in any medium or format, as long as you give appropriate credit to the original author(s) and the source, provide a link to the Creative Commons licence, and indicate if changes were made. The images or other third party material in this article are included in the article's Creative Commons licence, unless indicated otherwise in a credit line to the material. If material is not included in the article's Creative Commons licence and your intended use is not permitted by statutory regulation or exceeds the permitted use, you will need to obtain permission directly from the copyright holder. To view a copy of this licence, visit http://creativecommons.org/licenses/by/4.0/.

(C) The Author(s) 2021 